\title{
A rare case showing an enlarged level I lymph node as the first symptom due to papillary thyroid microcarcinoma
}

\author{
Wei Ding ${ }^{1}$, Shuli Zhao ${ }^{2}$, Zhimimg $\mathrm{Ma}^{3}$, Wei Lin ${ }^{1}$, Shuang $\mathrm{Li}^{4}$, Hong Zhang ${ }^{1}$ \\ ${ }^{1}$ Department of Thyroid Surgery, ${ }^{2}$ Department of Operation Room, ${ }^{3}$ Department of Gastrointestinal Nutrition and Hernia Surgery, ${ }^{4}$ Department of \\ Ear-Nose-Throat Surgery, The Second Hospital of Jilin University, Changchun 130041, China \\ Correspondence to: Shuang Li, MD, PhD. Department of Ear-Nose-Throat Surgery, The Second Hospital of Jilin University, Changchun 130041, \\ China. Email: 2570051137@qq.com; Hong Zhang, MD, PhD. Professor, Department of Thyroid Surgery, The Second Hospital of Jilin University, \\ Changchun 130041, China. Email: 2570051137@qq.com.
}

\begin{abstract}
As the high-pace development of sonography in recent years, the incidence of papillary thyroid carcinoma (PTC) has increased sharply worldwide, especially papillary thyroid microcarcinoma (PTMC). As we all know, PTMC is an indolent tumor, and level I lymph nodes (LNs) at a higher region relatively, so the majority of PTMCs admitted to surgery before the level I LNs metastasis. It is rare to see PTMC with level I LNs metastasis. At best of our known, there is only one Chinese research mentioned it but without a description of it. In this case, we reported a 30-year-old female who developed a recurrent submental cystic mass after being received treatment to remove a certain volume of light-yellow fluid. Pathology verified that mass in the submental area involved by PTMC. We consider that it may relate to age, multifocality, the diameter of PTMC, numbers of central compartment LNs, and "re-metastasis".
\end{abstract}

Keywords: Papillary thyroid microcarcinoma (PTMC); level I lymph node (level I LN); metastasis

Submitted Oct 16, 2019. Accepted for publication Apr 29, 2020.

doi: $10.21037 / g s-19-363$

View this article at: http://dx.doi.org/10.21037/gs-19-363

\section{Background}

The incidence of papillary thyroid carcinoma (PTC) has increased sharply worldwide, especially papillary thyroid microcarcinoma (PTMC), which due to applying sonography at a large scale in recent decades (1-3). PTMC tends to metastasize cervical lymph nodes (LNs), but level I LNs were rarely involved. We speculate that level I LNs at a higher position than other level LNs, PTMC need a long time to involve level I LNs because of its biological characteristics (2). In other words, people, who with level I LNs metastasis, are at an advanced tumor grade. Based on it, we consider that many patients received operation before level I LNs metastasis. That is why it is rare to see. However, we treated a rare case of a patient who shown an enlarged level I LN as the first symptom. This case suggested that we should consider of PTMC metastasis when a patient has a mass of neck.

\section{Case presentation}

The case is a 30 -year-old female with a submental cystic mass that had grown rapidly in the last 4 months. Treatment was performed to remove a certain volume of light yellow fluid 1 month ago, which made the mass significantly smaller. Unfortunately, the mass enlarged after. The patient referred to our hospital for a complete treatment.

Physical examination showed a $3.8 \mathrm{~cm}$ nodule in the submental region, which is hard and unmovable (Figure 1). In the area of level III, a hard-unmovable nodule measured $1.0 \times 0.5 \mathrm{~cm}$ was found. Cervical ultrasound examination showed multifocality in the thyroid, multiple enlarged LNs in level VI and bilateral neck, and a swollen LN on the level I area of the neck (Figure 2). Sonography interpreted as PTMC (both lobes) with metastasis to level I, level VI, level III of the left lateral neck, level II of the bilateral neck. Except for any enlarged LNs, multiple abnormal densities 


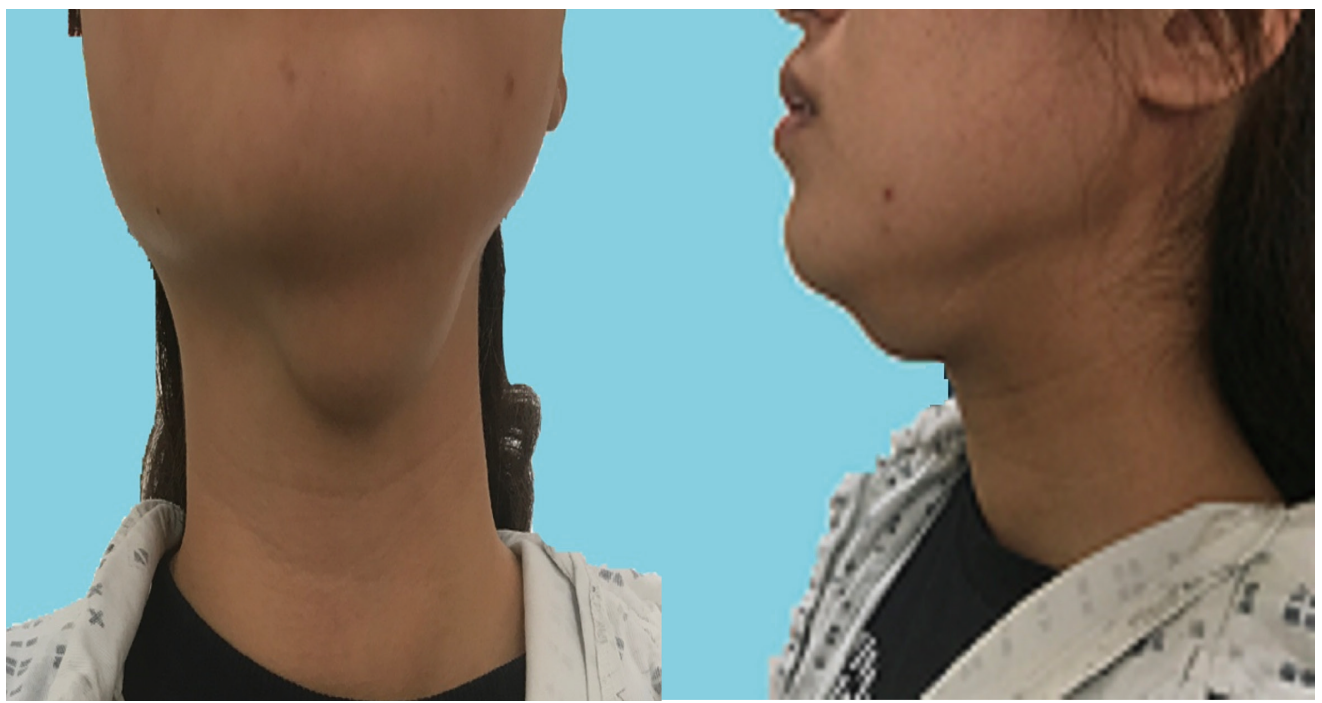

Figure 1 The nodule in the submental region.
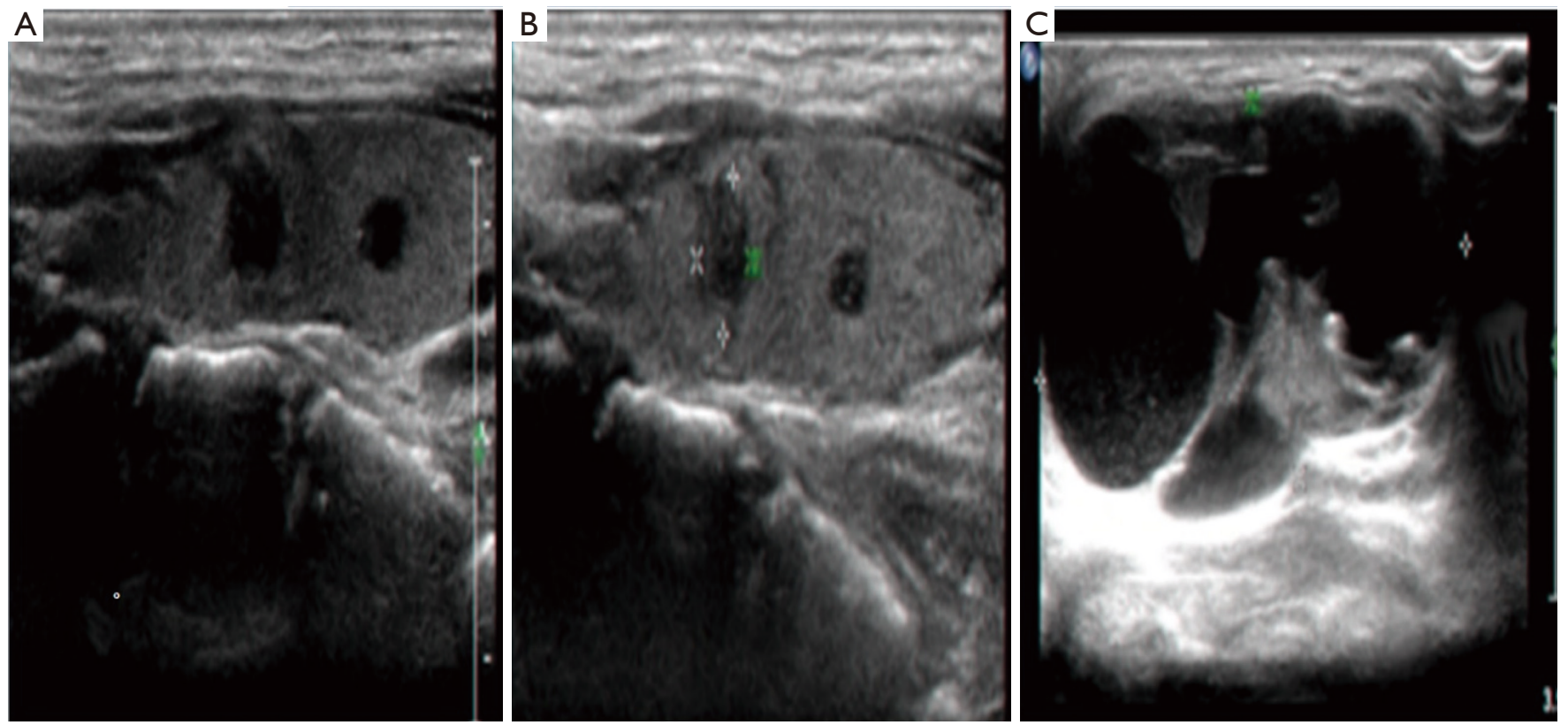

Figure 2 Neck sonography: (A) left lobe of thyroid; (B) right lobe of thyroid; (C) enlarged level I LN. LN, lymph node.

of the area in submental and anterior of hyoid bone were shown by computer tomography (CT) scanning Figure 3). Total thyroidectomy combined with a dissection of level VI LNs was performed under general anesthetic. During surgery, the appearance of the thyroid gland was normal, and surrounding tissues were not involved. Intraoperative pathological assessment of thyroid specimens and level
VI LNs shown multifocal PTMC with level VI LNs metastasis. Two focalities in the left lobe with 0.6 and $0.5 \mathrm{~cm}$ in diameter. One focality in the right lobe with $0.3 \mathrm{~cm}$ in diameter. Left level VI LNs metastasis (8/8), right level VI LNs metastasis (1/8). Combined with imaging assessments, bilateral LNs dissection was operated for further treatment. A $4.5 \times 2.0 \mathrm{~cm}$ necrotic $\mathrm{LN}$ was found 

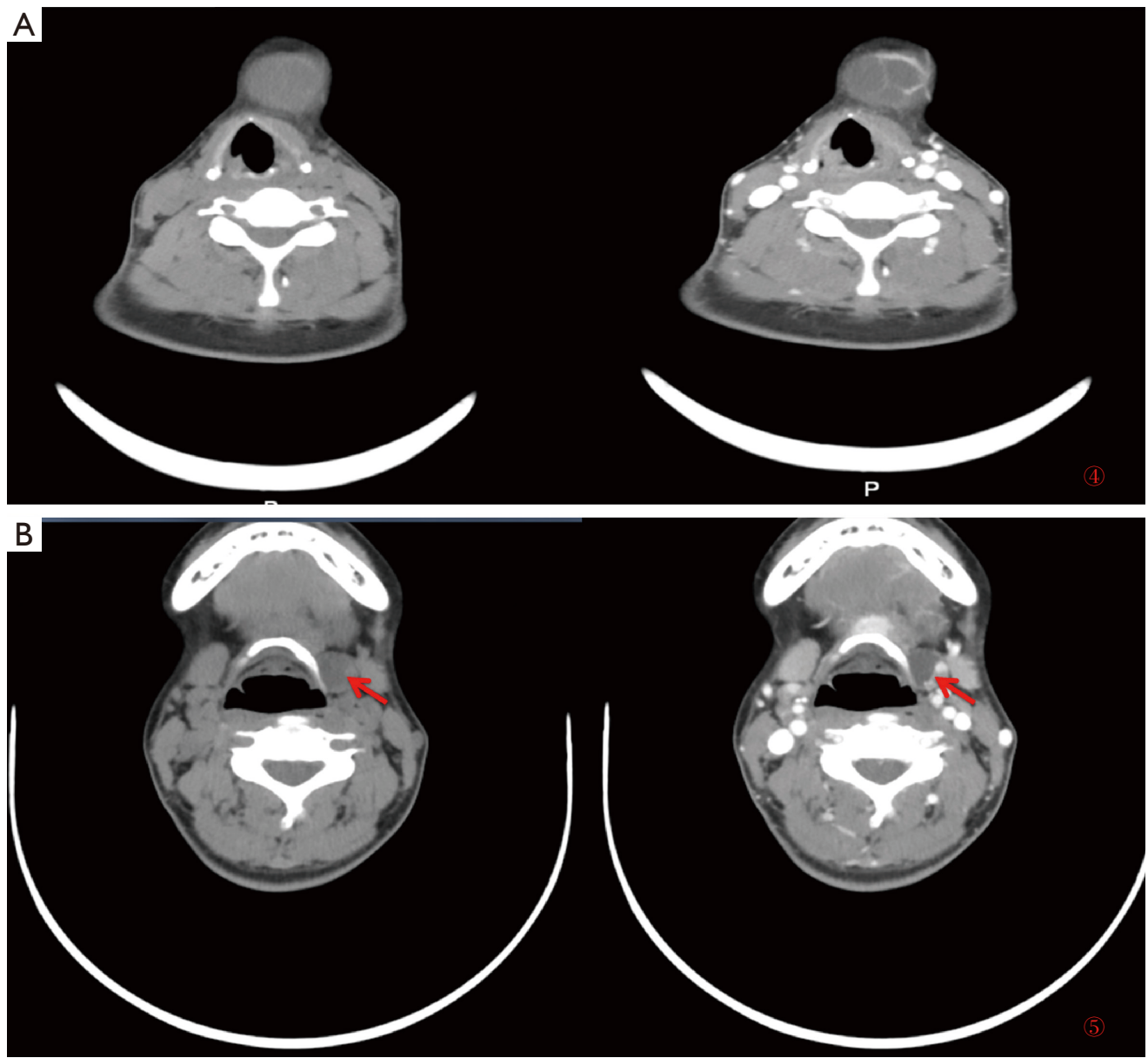

Figure 3 CT scanning: (A) enlarged level I LN; (B) a nodule in the anterior of the hyoid bone. CT, computer tomography; LN, lymph node.

in level I; a nodule was found in the anterior of hyoid measured $2.0 \times 1.0 \mathrm{~cm}$ (Figure 4 ). The postoperative pathological assessment revealed level I LN metastasis (1/1). In the left neck, level II-IV and part of level V LNs metastasis (6/35). In the right neck, level II-IV and part of $\mathrm{V}$ lymph metastasis (0/34). PTMC involved the tissue in the anterior of the hyoid. One month after the operation, static thyroid imaging and salivary gland imaging showed no abnormality. The patient admitted to ${ }^{131} \mathrm{I}$ therapy. Three months after the operation, there was no recurrent focality.

\section{Discussion}

With a significantly increased incidence of PTMC, thyroid carcinoma is surmised to become the $3^{\text {rd }}$ most frequent cancer in women by 2019 (1). PTMC characterized by less aggressive and good prognosis, but it also tends to metastasize cervical LNs. The LNs in the central compartment (level VI) at the highest risk being metastasized among cervical LNs. Sometimes lateral LNs can involve, especially these in level III $(2,3)$. However, it is extremely rare to see level I LNs metastasis (4). We speculate that level I LNs at a higher position than other level LNs, PTMC need a long time to involve level I LNs because of its biological characteristics. In other words, people, who with level I LNs metastasis, are at an advanced tumor grade. Based on it, we consider that many patients received operation before level I LNs metastasis. That is why it is rare to see. However, in this case, the patient with level I LNs at an early stage, we considered that it related to some factors when reviewing literature.

(I) Age. Age is a crucial factor in the prognosis of PTMC according to Thyroid Carcinoma Staging published by the American Joint Committee on 


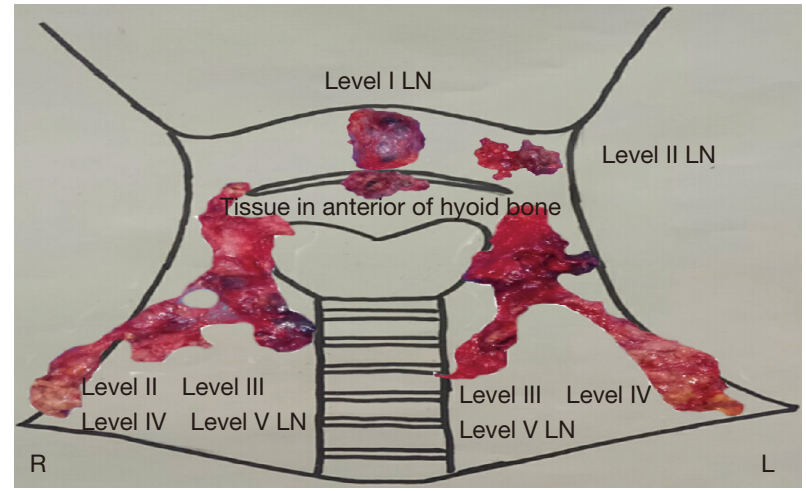

Figure 4 LNs in level I, bilateral level II-IV and part of level V, the tissue in the anterior of the hyoid. LN, lymph node.

Cancer (AJCC). Many studies put forward that patients with initial therapy at a younger age are more likely to metastasize to lateral neck LNs than those who are elder (4-7). So, we considered that this patient's age was a risk factor for level I LN metastasis.

(II) Multifocality. It is a sign of aggression of PTMC when a show as multifocality. Recent research showed that multifocality is an independent risk factor of PTMC with lateral neck LNs metastasis (7-10). In other words, PTMC with multifocality can metastasize to a further region. So, we speculated that multifocality in the left lobe might be one reason for level I LN metastasis.

(III) The number of the central compartment LNs (level VI), there was a positive relationship between level VI LNs metastasis and lateral neck LNs metastasis. In other words, the more positive level VI LNs revealed by intraoperative pathological assessment, the higher the risk of cervical LNs metastasis (11-13). In this case, it is possible that numbers of level VI LNs related to level I LNs metastasis.

(IV) "Re-metastasis". The focality, at the anterior of the hyoid bone, is a metastatic PTMC proved by postoperative pathology. This focality is of high invasion as the same as the first thyroid tumor. In general, distant metastasis of PTMC related to the site of focality. In other words, LNs or tissues near the thyroid are more vulnerable. It is why that PTMC tends to metastasize to the central compartment but rare to level I LNs. We supposed that the focality of the thyroid glands invaded into the tissue in the anterior of hyoid bone at first, and then the tissue as an "initial tumor" to metastasize to level I LN. We called this process re-metastasis.

We cannot deny that new cases of PTMC are increasing sharply every year worldwide. The newest edition of the American Thyroid Association (ATA) Guidelines suggests that closely follow-up has priority over surgery when PTMC with minimal risk of recurrence. It based on a low mortality rate and metastasis rate in the United States. However, many Chinese surgeons have found that Chinese patients' LNs metastasis rate and mortality rate are higher than American patients. In this case, the patient has PTMC but had metastasized to level I LN before she was referred to the hospital. In some ways, it may be the things that we need to think about whether ATA guidelines suit Chinese PTMC patients or not.

According to intraoperative pathological assessment, there is only one metastasis LN in level VI, and it means that the patient's right lateral neck $\mathrm{LN}$ has a minimal risk of metastasis. It still needs a long-term follow-up to proof that the patient who is like this case can receive help from bilateral LNs dissection.

Besides that, we should pay attention to the patient who has a mass of neck, and it may come from a PTMC metastasis.

\section{Acknowledgments}

Funding: None.

\section{Footnote}

Conflicts of Interest: All authors have completed the ICMJE uniform disclosure form (available at http://dx.doi. org/10.21037/gs-19-363). The authors have no conflicts of interest to declare.

Ethical Statement: The authors are accountable for all aspects of the work in ensuring that questions related to the accuracy or integrity of any part of the work are appropriately investigated and resolved. Ethical approval was given by the medical ethics committee of the Second Hospital of Jilin University with the following reference number: No. 125, 2017. The report follows the principles of medical ethics, tests, and treatments in this report that are consented by the patient and her families. Confidentiality of the patient's personal information to protect the patient's 
privacy. Written informed consent was obtained from the patient for publication of this case report and any accompanying images.

Open Access Statement: This is an Open Access article distributed in accordance with the Creative Commons Attribution-NonCommercial-NoDerivs 4.0 International License (CC BY-NC-ND 4.0), which permits the noncommercial replication and distribution of the article with the strict proviso that no changes or edits are made and the original work is properly cited (including links to both the formal publication through the relevant DOI and the license). See: https://creativecommons.org/licenses/by-nc-nd/4.0/.

\section{References}

1. Aschebrook-Kilfoy B, Kaplan EL, Chiu BC, et al. The acceleration in papillary thyroid cancer incidence rates is similar among racial and ethnic groups in the United State. Ann Surg Oncol 2013;20:2746-53.

2. Merdad M, Eskander A, Kroeker T, et al. Metastatic papillary thyroid cancer with lateral neck disease: pattern of spread by level. Head Neck 2013;35:1439-42.

3. Wang Y, Guan Q, Xiang J, et al. Clinicopathologic features and prognostic factors of diffuse sclerosing variant of papillary thyroid carcinoma: a population-based analysis. Transl Cancer Res 2018;7:695-705.

4. Lombardi CP, Bellantone R, De Crea C, et al. Papillary thyroid microcarcinoma: extrathyroidal extension, lymph node metastases, and risk factors for recurrence in a high prevalence of goiter area. World J Surg.2010;34:1214-21.

5. Cho JK, Kim JY, Jeong CY, et al. Clinical features and

Cite this article as: Ding W, Zhao S, Ma Z, Lin W, Li S, Zhang H. A rare case showing an enlarged level I lymph node as the first symptom due to papillary thyroid microcarcinoma. Gland Surg 2020;9(3):797-801. doi: 10.21037/gs-19-363 prognostic factors in papillary thyroid microcarcinoma depends on age. J Korean Surg Soc 2012;82:281-7.

6. Qu N, Zhang L, Ji QH, et al.Risk factors for central compartment lymph node metastasis in papillary thyroid microcarcinoma: a meta-analysis. World J Surg 2015;39:2459-70.

7. Zhao Q, Ming J, Liu C, et al. Multifocality and total tumor diameter predict central neck lymph node metastases in papillary thyroid microcarcinoma. Ann Surg Oncol2013;20:746-52.

8. So YK, Son YL, Hong SD, et al. Subclinical lymph node metastasis in papillary thyroid microcarcinoma: a study of 551 resections. Surgery 2010;148:526-31.

9. Kim KE, Kim EK, Yoon JH, et al. Preoperative prediction of central lymph node metastasis in thyroid papillary microcarcinoma using clinicopathologic and sonographic features. World J Surg 2013;37:385-91.

10. Zhang L, Wei WJ, Ji QH, et al. Risk factors for neck nodal metastasis in papillary thyroid microcarcinoma: a study of 1066 patients. J Clin Endocrinol Metab 2012;97:1250-7.

11. Lee YS, Lim YS, Lee JC, et al. Clinical implication of the number of central lymph node metastasis in papillary thyroid carcinoma: preliminary report. World J Surg 2010;34:2558-63.

12. Rajeev P, Ahmed S, Ezzat TM, et al. The number of positive lymph nodes in the central compartment has prognostic impact in papillary thyroid cancer. Langenbecks Arch Surg 2013;398:377-82.

13. Xiao GZ, Gao L. Central lymph node metastasis: is it a reliable indicator of lateral node involvement in papillary thyroid carcinoma? World J Surg 2010;34:237-41. 